\title{
A Paradigm Shift in Primary Open Angle Glaucoma
}

\section{BY MARK ELTIS, OD, FAAO}

\section{Introduction}

G laucoma is a chronic irreJersible neurodegenerative disease characterized by destructive changes in the optic nerve head and retinal nerve fibre layer due to loss of retinal ganglion cells and their axons. ${ }^{1-14}$

For years glaucoma was considered to be a disease of ocular hypertension (greater than $21 \mathrm{mmHg}$ ). ${ }^{15-17}$ Over the last decade the disease has been redefined as an optic neuropathy with field loss resulting from IOP unacceptably high for the optic nerve head. ${ }^{6,8,18-21}$ Therefore, the simplistic cut-off point of $21 \mathrm{mmHg}$ seems outdated and invalid. $8,19,22$

Despite the prevalence of glaucoma, a universal definition of the disease is still absent. ${ }^{23-27}$

Glaucoma is a leading cause of blindness worldwide and the leading cause of vision loss in the United States. ${ }^{4,6,15,16,25}$ Approximately $2 \%$ of the U.S. population older than 40 have glaucoma and, with the aging of the population, the number of patients with the disease is expected to increase. ${ }^{2,15,16,25}$ Glaucomatous neuropathy is generally an insidious disease with no symptoms until the advanced stages. ${ }^{2,14,28}$ There is a large body of evidence to suggest that an estimated $50 \%$ of those with glaucoma have not been diagnosed. ${ }^{6,28}$

Primary open angle glaucoma (POAG), the most common glaucoma $^{29,30}$ (representing $90-95 \%$ of

\section{ABSTRACT}

The definition, diagnosis and management of glaucoma have changed radically over the last decade. This paper reviews recent advances in primary open angle glaucoma and how they were applied to the case of a 68-year-old patient.

Key words: pachymetry, neurodegenerative, prostaglandin analogue

cases), ${ }^{2}$ is defined by optic neuropathy in the absence of an identifiable secondary cause. .,2, $, 6,21_{\text {POAG }}$ is a bilateral condition, but disease progression may be asymmetric., 2,16 POAG can be divided into high tension glaucoma (HTG) and normal tension glaucoma (NTG). ${ }^{30-32}$

Although it is frequently accompanied by increased IOP, POAG can exist in patients with normal IOP., ${ }^{2,15}$ Up to $50 \%$ of patients with glaucoma never have an IOP above the statistical norm. ${ }^{22,31,33}$ Therefore, IOP alone is not reliable for glaucoma screening. ${ }^{30,33}$ However, IOP remains the only modifiable risk factor. ${ }^{12,22}$ Early detection is critical to prevent permanent structural damage and irreversible vision loss. $2,3,28,34$ Over the past decade, the diagnosis and management of glaucoma have changed dramatically. ${ }^{15,16}$ Numerous advances, such as the diagnostic role of pachymetry, ${ }^{35,36}$ imaging devices, ${ }^{37-41}$ advanced perimeters ${ }^{42-44}$ and new therapeutic options, ${ }^{45-47}$ have revolutionized the diagnosis and management of the disease. ${ }^{3}$

\section{Case Report}

A 68-year-old female retiree of Hispanic descent presented to our office on July 8, 2009 for a routine exam. She was not satisfied with the quality of her old glasses and wanted to update her prescription. She was not taking any medication and had no history of health problems either in general or specifically of the eye. The patient had neither worn contact lenses nor had any corrective surgery. The patient had no known allergies and was not aware of health conditions in her family, was neither a smoker nor a drinker and did not engage in recreational drug use.

Presenting distance visual acuities through her glasses $(+3.25-2.00$ $\times 065 \mathrm{OD}$ and $+3.25-2.00 \times 120$ OS) were 20/40 OD, 20/20 OS and $20 / 20$ OU. She was wearing progressive addition lenses, and her vision through her +2.25 reading addition was 20/30 OD, 20/20 OS and 20/20 OU (40 cm working distance).

Extraocular muscles were unrestricted in all gazes. Pupils were round and reactive to light and accommodation with negative Marcus Gunn. Confrontation visual fields were full to finger count in both eyes, and near point of convergence was $8 \mathrm{~cm}$. Cover test was ortho in the distance and at near.

Subjective refraction was OD $+2.25-2.00 \times 06120 / 25$ and OS $+3.25-2.00 \times 12020 / 20$. Her new add was +2.50 . 
Slit lamp examination revealed normal lids and lashes. The conjunctivas of both eyes were clear. Both corneas had arcus senilis present but were otherwise clear and did not stain with fluorescein. Irises were brown, and transillumination was absent in both eyes. Both crystalline lenses had grade 1 cortical spoking and nuclear sclerosis. (There was no pseudoexfoliation in either eye.) Anterior chambers were without either cells or flare and were estimated by Van Herick grading to be grade 3 in both eyes. Intraocular pressure was $16 \mathrm{mmHg}$ in both eyes at 3:30 p.m. using Perkins applanation tonometry. Dilated fundus examination was performed. Cup-to-disc ratios were 0.8/0.8 OD and 0.7/0.7 OS. The arterial-venous ratio was $2 / 3$ in $\mathrm{OU}$, and the retinal vessels appeared normal. Maculas were clear in both eyes although a foveal reflex was absent. There were neither holes, tears nor instances of retinal detachment in either eye. The patient was counselled that a glaucoma work-up would be in order. She said there was no family history of glaucoma, but she was asked to inquire further to be sure. The patient was asked to book a follow-up for a check of IOP, for gonioscopy and for another look at the fundi. The patient declined to be sent for visual field tests, pachymetry, OCT and HRT. She was also informed about the early cataracts and arcus. (The patient said she had just had her cholesterol checked and that it was normal.)

\section{Follow-up \#1}

After initially declining all further testing and then taking an extended vacation overseas, the patient returned for a follow-up on September 19, 2009. She stated that her brother was in fact being treated for glaucoma. Presenting distance visual acuities through her glasses $(+3.25-2.00 \times 065 \mathrm{OD}$ and +3.25 $-2.00 \times 120$ OS) were 20/40 OD, 20/20 OS and 20/20 OU. Intraocular pressure was $16 \mathrm{mmHg}$ in both eyes at 1:15 p.m. using Perkins applanation tonometry. Gonioscopy (Goldmann) was performed and did not reveal any pathology. Scleral spur was identified in all four quadrants of both eyes. Anterior chambers were without either cells or flare. Dilated fundus examination revealed that cup-to-disc ratios were $0.8 / 0.8$ OD and $0.7 / 0.7$ OS. There was no indication of either optic nerve drusen or nerve pallor in either eye. The arterial-venous ratio was $2 / 3$ in $\mathrm{OU}$, and the retinal vessels appeared normal. Maculas were clear in both eyes although a foveal reflex was absent. The patient was scheduled for pachymetry, OCT, HRT and a visual field test.

\section{Follow-up \#2}

The patient rescheduled her followup several times but finally had testing done on January 6, 2010.

Visual field testing showed a large inferior arcuate defect $\mathrm{OU}$ which corresponded to the NFL superior defect on both the OCT and HRT. The visual field test was very reliable, and GHT was outside normal limits in both eyes. The signal strength of the OCT was a less than ideal 6 in each eye, probably due to media opacities. Central corneal thickness (CCT) was $510 \mu \mathrm{m}$ OD and $515 \mu \mathrm{m}$ OS. The patient was informed that she was a glaucoma suspect and was scheduled for a consult with a glaucoma specialist.

\section{Follow-up \#3}

The patient failed to appear for her follow-up but finally returned for a visual field at the glaucoma specialist's office on July 29, 2010, prior to the glaucoma consult. A repeatable arcuate defect was confirmed in each eye. At that visit the patient's corrected visual acuity was $20 / 40^{+2}$ OD and 20/30 OS. Her IOP was 16 $\mathrm{mmHg}$ in each eye at 11 a.m. using Goldmann tonometry.

\section{Follow-up \#4}

The patient was then seen by the glaucoma specialist on August 3, 2010. IOP at 12 p.m. was $16 \mathrm{mmHg}$ OU. Gonioscopy was performed, and angles were found to be "open" in both eyes. Cup-to-disc ratios were 0.8/0.6 OD and 0.8/0.6 OS and a "superior rim notch" was noted OU. OCT was repeated and confirmed superior NFL damage in both eyes. An "arcuate defect" in each eye was identified based on the 24-2 Humphrey visual field test (performed July 29, 2010), which was consistent with the area of damage on OCT.

The following differential diagnosis was considered in this case:

\section{Physiological cupping has} neither field loss, progression of cupping nor damage to NFL. ${ }^{18,31}$ Although cupping may be large, the neuroretinal rim is healthy. ${ }^{10}$

\section{Congenital anomaly of the disc} is characterized by non-progressive visual field defects. ${ }^{10,31}$ Examples include tilted discs, colobomas and optic nerve pits. ${ }^{18}$ 


\begin{tabular}{|c|c|c|c|c|c|}
\hline Name: & & & $O D$ & os & ZEISX \\
\hline ID: & & Exam Date: & $8 / 3 / 2010$ & $8 / 3 / 2010$ & \\
\hline DOB: & & Exam Time: & $9: 18 \mathrm{AM}$ & 9:19 AM & \\
\hline Gender: & Female & Technician: & & & \\
\hline Doctor: & & Signal Strength: & $8 / 10$ & $6 / 10$ & \\
\hline
\end{tabular}

\section{\begin{tabular}{ll|l|} 
RNFL Thickness Analysis:Optic Disc Cube $200 \times 200$ & OD & OS \\
\hline
\end{tabular}}

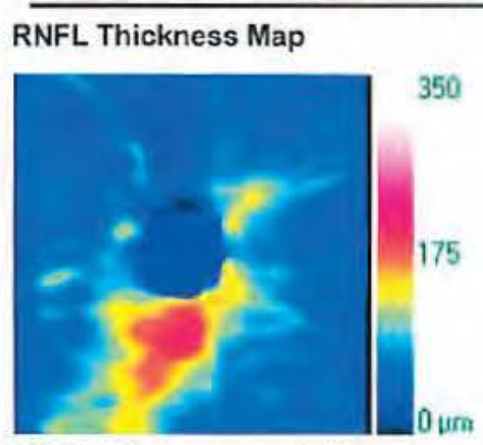

RNFL Thickness Deviation

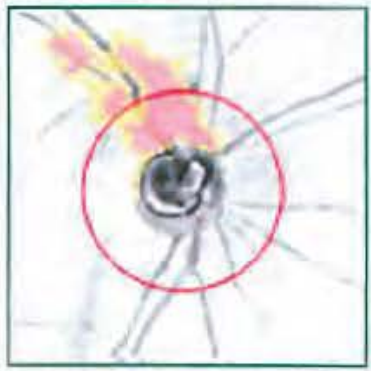

Offset $\{-0.12 ;-0.03) \mathrm{mm}$

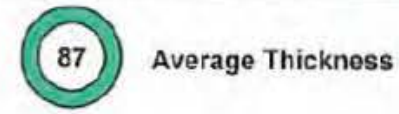

65

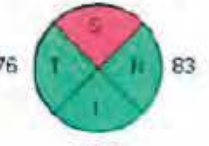

124

$63 \quad 52 \quad 81$

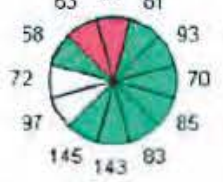

$\mu \mathrm{m} \longrightarrow \mathrm{OD} \ldots . . . . \mathrm{O}$

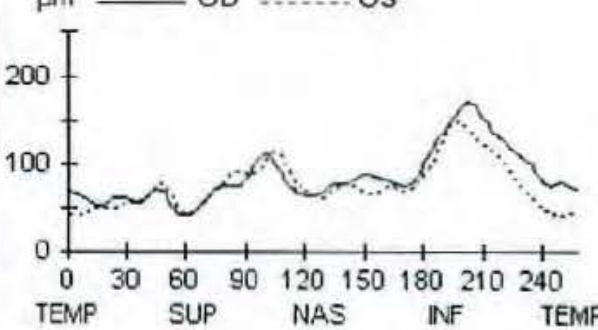

RNFL. TSNIT Normative Data

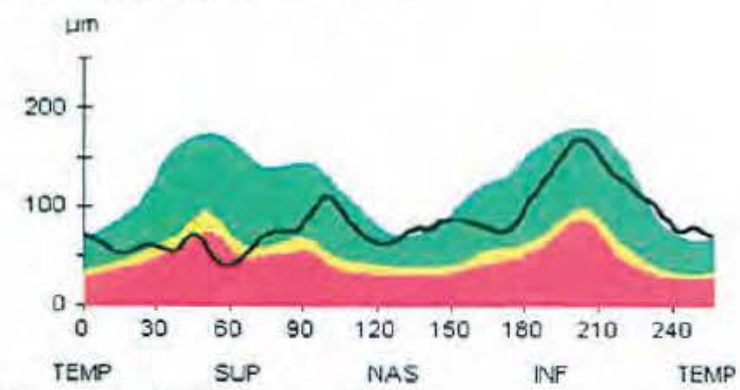

Extracted RNFL Tomogram

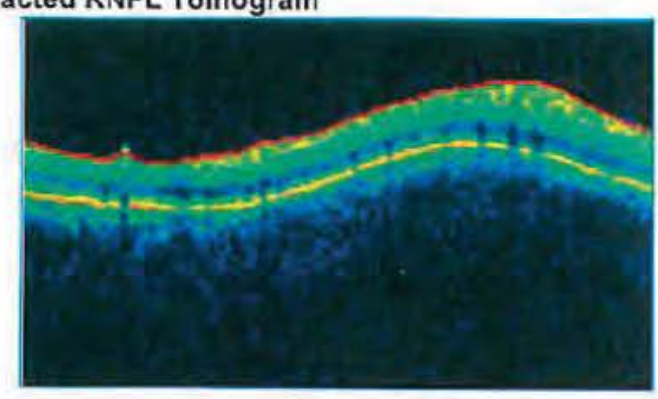

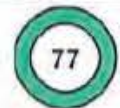

69

83

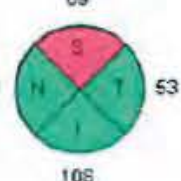

83

Clock

Hours
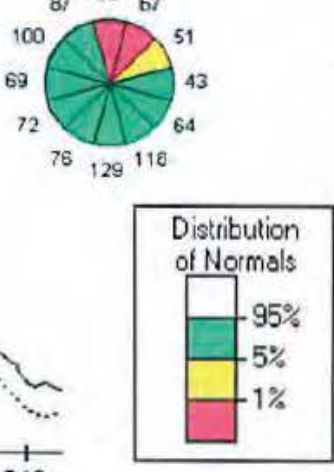

Symmetry
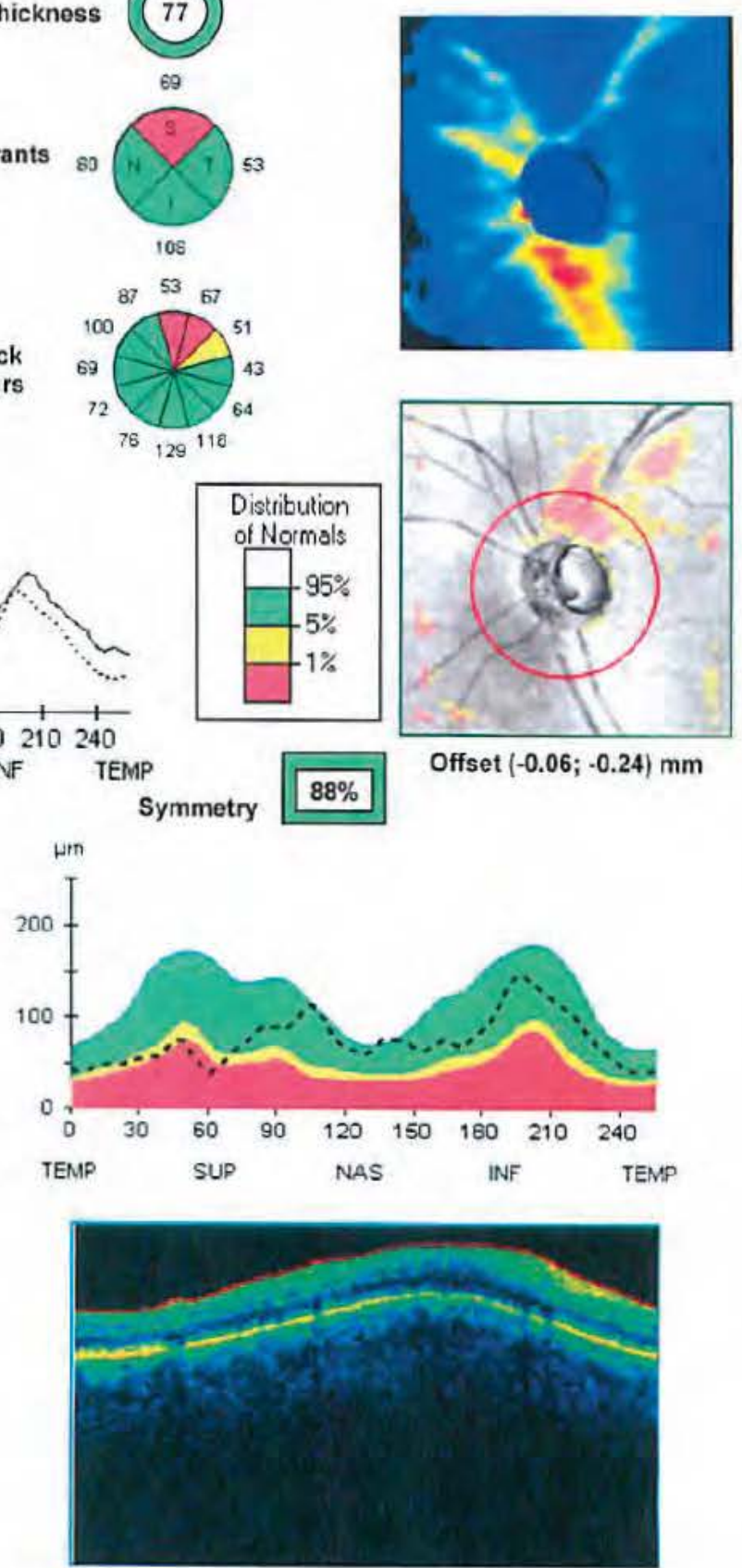

Figure 1 - OCT showing superior RNFL damage in both eyes. Exam date: August 3, 2010. 


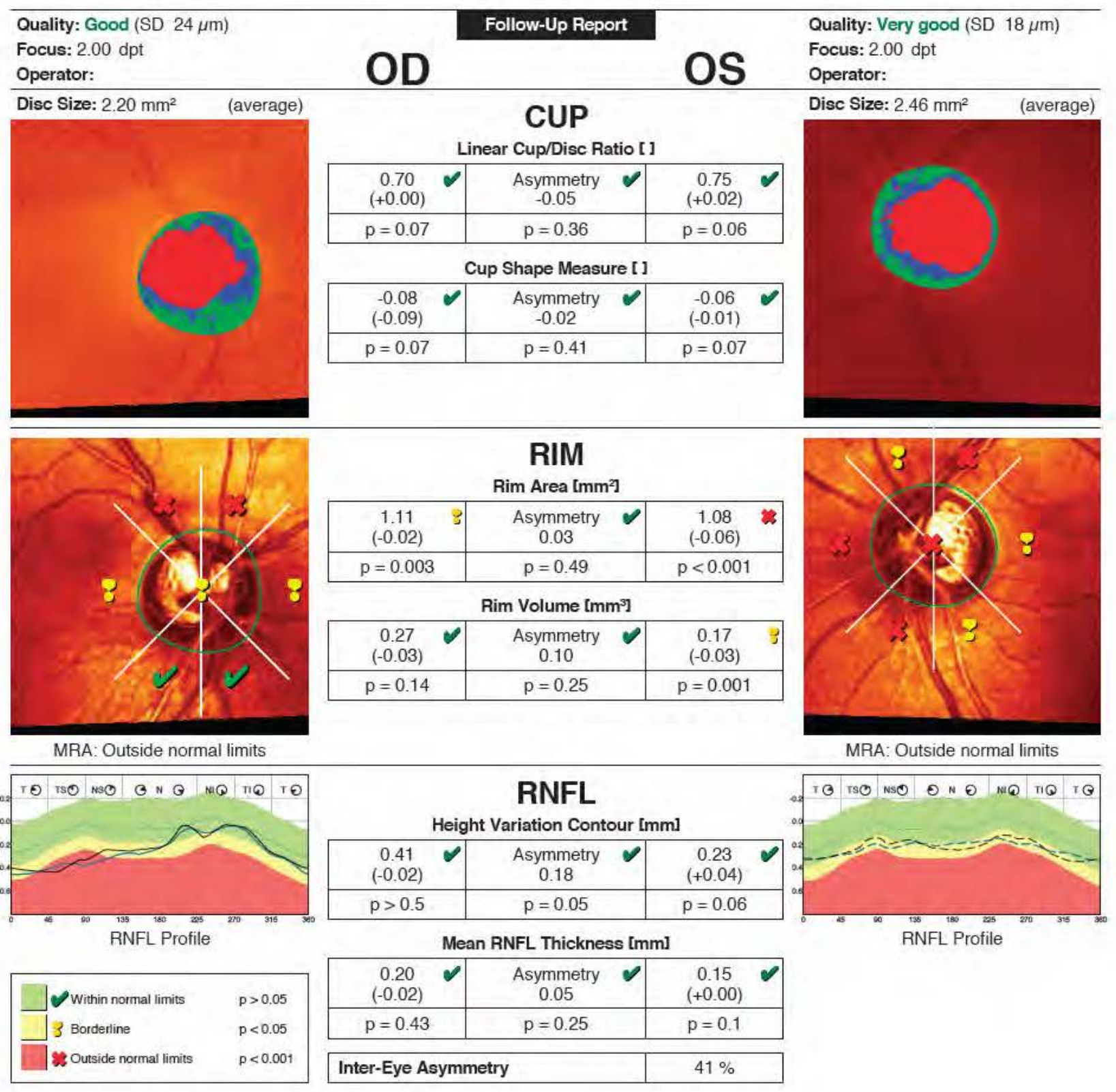

Figure 2-HRT. Exam date: October 13, 2010.

3. Optic atrophy is characterized by more optic nerve pallor than cupping. ${ }^{10}$ There may be a reduction in the small vessels on the disc surface (Kestenbaum sign). ${ }^{13}$ Central vision is generally decreased. ${ }^{18}$ The numerous causes of optic atrophy include tumours and either vascular or degenerative disease. ${ }^{18}$ Visual field defects that respect the vertical midline are typical of intracranial lesions. ${ }^{13}$

4. Optic nerve drusen are hyalinelike, may be either superficial or buried ${ }^{13}$ and are associated with retinitis pigmentosa. ${ }^{10} \mathrm{With}$ drusen, an absence of cupping may be visible. ${ }^{18,31}$ Lesions can be seen on B-scan ${ }^{18}$ and demonstrate autofluorecsence with fluorescein filters through a fundus camera. ${ }^{10}$ Drusen may cause arcuate scotomas to develop. ${ }^{10}$ Several $\mathrm{ONH}$ drusen may be visible as 
elevated chunky refractile nodules. ${ }^{10}$ Buried drusen obscure the physiological cup ${ }^{18,31}$ and exposed drusen emerge during the teen years and are visible. ${ }^{13}$

\section{POAG (divided into NTG} and HTG) is optic neuropathy with IOP too high for the optic nerve. ${ }^{30-32}$ POAG by definition has an open anterior chamber (confirmed with gonioscopy) and no identifiable cause for the elevated IOP. ${ }^{16}$ Cupping, notching of the optic nerve along with corresponding visual field loss is present. ${ }^{13}$ POAG is generally bilateral but may be asymetric. ${ }^{18} \mathrm{Visual}_{\text {field }}$ defects cross the vertical midline, and central scotomas are absent early in the disease. ${ }^{31}$

\section{Secondary glaucoma is a root} cause for the elevated IOP. Either optic nerve damage or field loss must be present. Causes include pigment dispersion syndrome, pseudoexfoliation of the lens, steroid use and trauma. ${ }^{16}$ Secondary glaucoma can be ruled out through case history and SLE examination with gonioscopy. ${ }^{10,16}$

Our patient had nerve fibre damage with corresponding areas of field loss. The damage was bilateral and consistent with glaucomatous loss. Her central corneal thickness was thin in each eye. There was no history of steroid use or previous glaucoma episodes. No optic nerve drusen were seen, and the cupping was large rather than obliterated. Pallor of the optic nerves was also absent.

Neuro-opthalmological examination did not seem indicated ${ }^{31}$ considering the bilateral cupping and the type of visual field loss

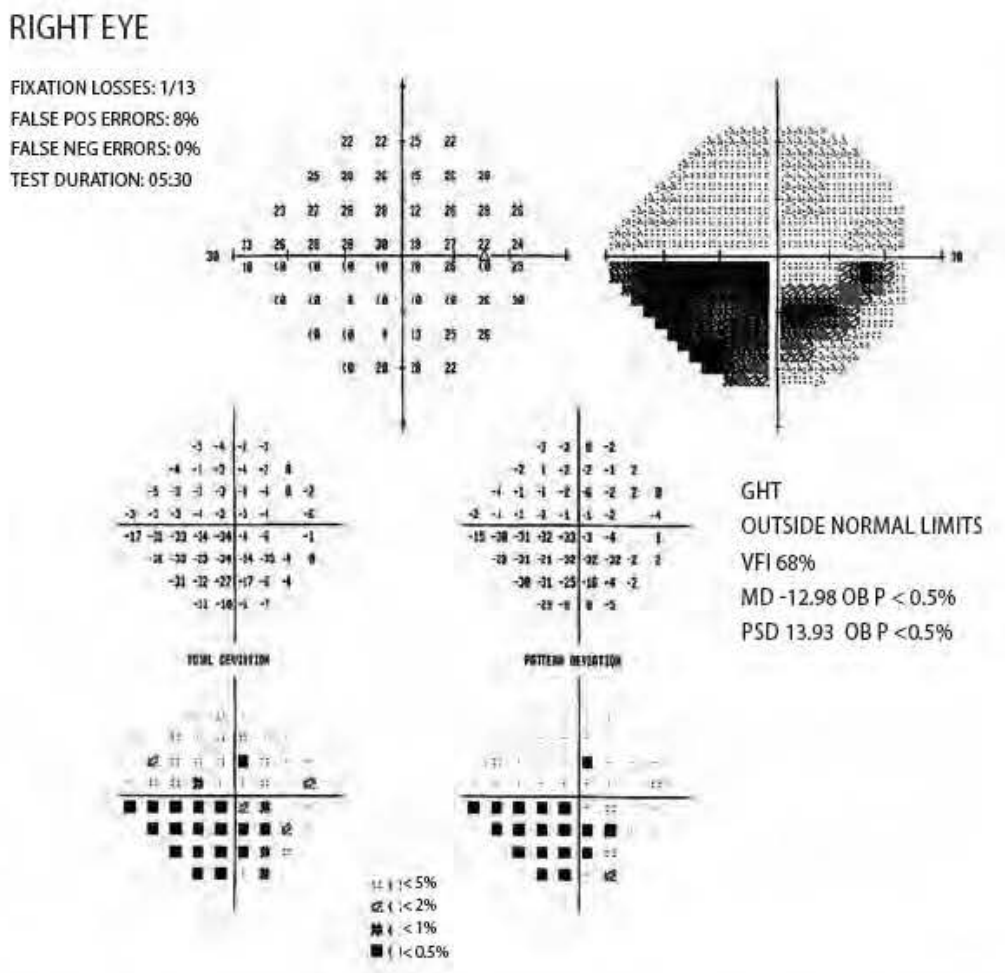

\section{LEFT EYE}

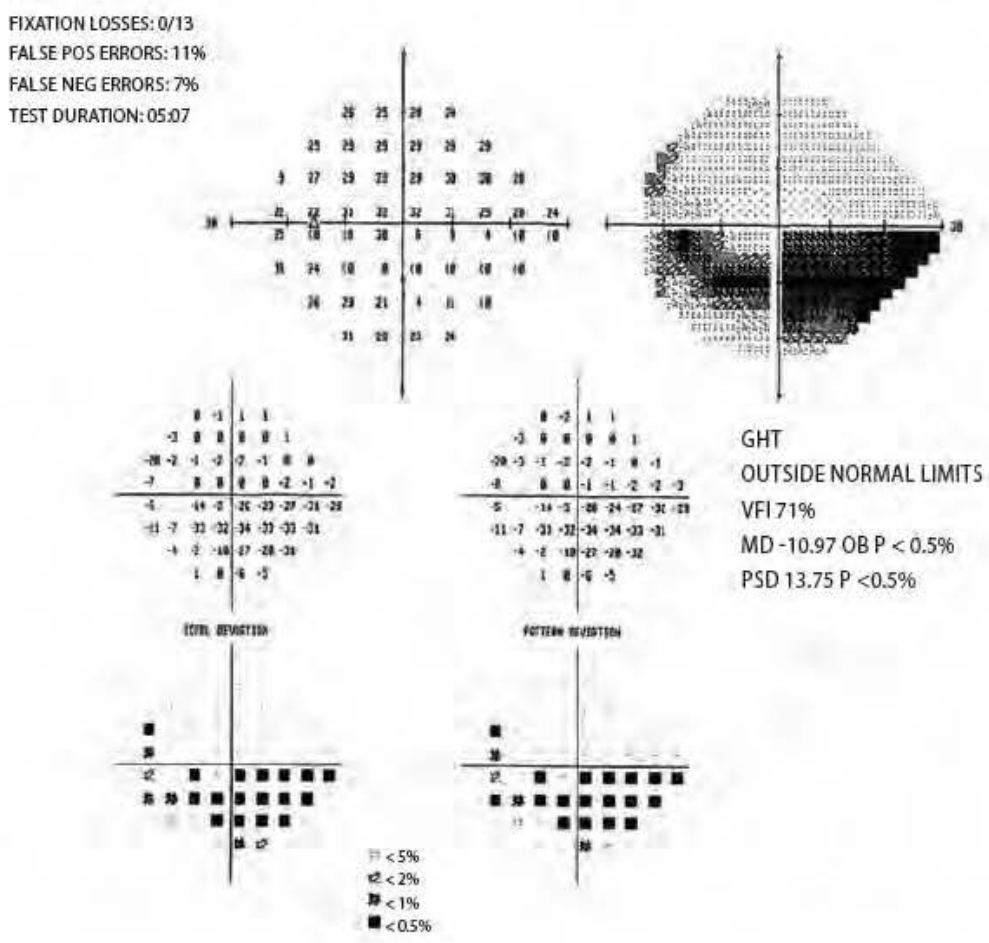

Figure 3-24-2 Humphrey visual field test. Exam date: October 13, 2010. 
which crossed the vertical midline. Gonioscopy and slit lamp examination did not reveal a secondary cause for the suspected glaucoma. Therefore, the tentative diagnosis was primary open angle glaucoma likely of the NTG subgroup because the pressure reading was within the statistical norm. However, it is possible that, if multiple readings of IOP had been taken over a longer period and particularly at night, there may either have been times when the patient's IOP was elevated above $21 \mathrm{mmHg}$ or have been large diurnal fluctuations.

The glaucoma specialist prescribed Travatan Z: 1 drop once per day OU for our patient. She was to return for a follow-up in two months.

\section{Follow-Up \#5}

The patient repeated HRT and 24-2 visual fields on October 13, 2010 at the glaucoma specialist's office. Her presenting visual acuities were $20 / 30^{-3}$ OD and $20 / 25^{-2}$ OS with glasses. IOP was $10 \mathrm{mmHg} O D$ and $14 \mathrm{mmHg}$ OS at 4:08 p.m. using Goldmann tonometry.

\section{Follow-Up \#6}

The patient was subsequently seen in the glaucoma specialist's office on October 19, 2010 for a consult. The patient's IOP was noted as $12 \mathrm{mmHg}$ in both eyes at 3 p.m. Pachymetry was $510 \mu \mathrm{m}$ OD and $515 \mu \mathrm{m}$ OS. "Adequate IOP control" was recorded in the assessment, and a follow-up appointment was scheduled for 6 months.

\section{Follow-Up \#7}

The patient was scheduled for follow-up with the glaucoma specialist on April 20, 2011. She did not appear for her appointment and did not return either my office's phone calls or the specialist's despite numerous attempts.

\section{Discussion}

\section{Epidemiology}

Glaucoma affects 80 million people ${ }^{14}$ and is the second leading cause of blindness worldwide. ${ }^{21,48}$ The prevalence of glaucoma varies widely around the globe, with the lowest rate found among the First Nations of Alaska $(0.006 \%)$ and the highest among Caribbean people of African ancestry $(7.1-8.8 \%) .^{49}$

Two million people have glaucoma in the U.S., ${ }^{2}$ and the number is expected to rise significantly. ${ }^{4}$

Rates are higher among Latinos than among Caucasians, in comparison with whom African Americans are 3-4 times more at risk. ${ }^{16} \mathrm{POAG}$ is the leading cause of blindness in African Americans. ${ }^{2}$ Normal tension glaucoma rates (among those affected with glaucoma) appear highest in Japan. ${ }^{30}$

\section{Risk Factors}

Glaucoma is a group of optic neuropathies with multiple risk factors, ${ }^{21}$ including increased cup-to-disc ratio, thin central corneal thickness, either African or Hispanic descent and the presence of an optic disc haemorrhage. ${ }^{2,13,15,36}$ Elevated IOP remains the most significant risk factor for glaucomatous progression. ${ }^{1,17}$ Optic disc haemorrhage is critical since it may precede visual field loss and further optic nerve damage. ${ }^{16}$
Positive family history is also an important risk actor. ${ }^{2,13,36,49}$ The familial nature of glaucoma has been recognized for decades with up to $50 \%$ of patients having a positive family history. ${ }^{4}$ The prevalence of glaucoma increases significantly in those 60 and older. ${ }^{2}$

The roles of diabetes ${ }^{15,16}$ and hypertension in glaucoma remain unclear. ${ }^{50,51}$ Controversially, some recent studies suggest that, in patients with either pre-existing diabetes or cardiovascular disease, the daily use of cholesterol drugs such as statins may reduce the risk of POAG development. ${ }^{2}$

The relationship between glaucoma and myopia is also still under investigation, ${ }^{2,16}$ with some evidence pointing to an increased risk of glaucoma among myopes. ${ }^{19,36}$ The LALES study suggested an independent relationship between increased axial length and glaucoma due to weaker scleral support at the optic nerve. ${ }^{16}$ There may be an association between NTG and Raynaud's phenomenon, migraines and hypotension. ${ }^{10,31,39}$ There are no environmental factors that are definitively associated with POAG. ${ }^{49} \mathrm{IOP}$ remains the only known risk factor that can be modified to decrease chances of disease progression. ${ }^{2}$

\section{Genetic Factors}

Clearly, there are genetic factors related to glaucoma. ${ }^{10}$ Less clear is the full extent of the relationship; although some genes have been identified, over $90 \%$ remain a mystery. ${ }^{4}$ To date, 22 genetic loci have been linked to POAG, and 3 genes have been identified for POAG from the reported loci. ${ }^{21,30}$ Mabuchi 
et al recently concluded that SRBD1 gene polymorphism (a non-IOPrelated genetic factor) is associated with both development of NTG and HTG POAG. ${ }^{30}$ Genes and some environmental factors may affect the rate of ganglion cell apoptosis. ${ }^{49}$

\section{Which Changes Happen First: Structural Or Functional?}

Structural change precedes functional change in some glaucomatous eyes but follows it in others. ${ }^{16}$ That some ganglion cells may begin to malfunction before dying results in decreased sensitivity without structural change. ${ }^{3}$ In other cases, where ganglion cell redundancy is high, retinal nerve fibre layer (RNFL) loss may precede functional damage..$^{11,38,39,42}$ In the OHTS study, $60 \%$ of patients who converted to glaucoma had optic disc changes before field loss. ${ }^{23,35,36}$

Both OHTS and EGPS showed that, in many patients, structural defects discovered with stereo photos are detectable before repeatable SAP (standard automated perimetry) functional defects. ${ }^{3}$

When SAP damage is detected first, it may be that the patient started out with more structure available to be lost and therefore that the patient continues to be within the norm for structure. ${ }^{3}$ Functional glaucoma deficits can occur before structural ones, but, more commonly, structural damage occurs before functional. ${ }^{5,11}$ The exact mechanism of damage is not yet fully understood. ${ }^{7,23,52}$

Diagnosis and management of glaucoma are shifting from disease staging to evidence-based risk assessment of the patient. ${ }^{51}$ Risk factor calculators, such as the one that merged data from OHTS and
EGPS (http://ohts.wustl.edu/risk) to create an online risk calculator for clinicians, now help to predict a patient's individual risk of developing glaucoma in the next five years. ${ }^{53}$

Because retinal ganglion cell dysfunction and death characterize glaucoma, ${ }^{20,54}$ detection is based on identification of either abnormalities or changes to either the $\mathrm{ONH}$ or the RNFL, either structural or functional. ${ }^{26,54}$ As there is no true gold standard for glaucoma diagnosis, progressive optic neuropathy has been suggested as a definition for use alongside functional testing., ${ }^{3,23}$

A purely functional view of glaucoma may miss early glaucomatous patients. ${ }^{3}$ The disease may more comprehensively be defined according to both functional and structural criteria. ${ }^{26,27}$

\section{Neuroprotection in Glaucoma}

Recent studies suggest the involvement of the immune system in glaucoma, ${ }^{12,20}$ but the neuroprotective and neurodestructive potentialities of the immune system make its role unclear and sometimes contradictory. ${ }^{8,14}$ T-cell - mediated immune response which is initially neuroprotective but goes unchecked $^{12}$ may become autoimmune neurodegeneration. ${ }^{8}$

Damage to retinal ganglion cells could potentially be prevented by targeting the mechanism behind neuronal destruction. ${ }^{20}$ Neuroprotective agents used in neurodegenerative diseases such as ALS (amyotrophic lateral sclerosis) and Alzheimer's disease are currently being evaluated for use in glaucoma. ${ }^{14}$
Several antibodies have been associated with glaucoma but no definitive specific antibody has been identified to screen for glaucoma. ${ }^{8}$

A recent study found that BDNF (brain-derived neurotrophic factor), a polypeptide growthfactor known to develop and to preserve neurons, ${ }^{20}$ may be a useful biochemical marker for early detection of glaucoma. ${ }^{6} \mathrm{BDNF}$ crosses the blood-brain barrier and is found in lower concentrations in the tears of those with glaucoma. ${ }^{6}$ This may also become a reliable, time-efficient and costeffective method for the screening and management of the disease. ${ }^{6}$ Vaccinations which boost the immune system such as ones being used to enhance neuroprotection in multiple sclerosis may become a promising therapy for glaucoma. ${ }^{12}$

\section{Ocular Hypertension and High Tension Glaucoma}

Ocular hypertension $(\mathrm{OH})$ is IOP elevated above the normal range. ${ }^{13}$ $\mathrm{OH}$ can exist without causing either damage to the nerve or vision loss but is a key risk factor for glaucoma. ${ }^{1}$ If left untreated, roughly 1 in 10 patients with $\mathrm{OH}$ will develop glaucoma in 5 years. ${ }^{1}$ The only proven strategy to prevent POAG progression is the use of ocular hypotensives among people with $\mathrm{OH} .{ }^{16,36,49}$ The decreasing of IOP lowers the rate of conversion from $\mathrm{OH}$ to POAG and slows its progression. ${ }^{2,15,36,50}$

In a glaucoma suspect, optic nerve deterioration (confirmed by either stereophoto, nerve fibre analysis or visual field glaucomatous changes) should be considered to indicate a conversion to POAG. ${ }^{54}$ 
This is why the establishment of a baseline through photography, imaging and fields is critical. ${ }^{16}$

\section{Normal Tension Glaucoma}

Normal tension glaucoma (NTG) is a form of POAG ${ }^{10,13,18}$ where optic nerve damage and corresponding visual field defects occur ${ }^{29,30}$ despite apparently normal IOP. ${ }^{55}$ With NTG, the etiological trigger - the pathogenic process - simply takes place at a lower IOP ${ }^{31,33}$ Whenever IOP in the individual is high enough to initiate the disease process, the pathophysiologic steps are the same as in HTG. ${ }^{31}$ Chinese glaucoma specialists reached a consensus in 2008 that NTG is a subtype of POAG and the dividing line was IOP greater than $21 \mathrm{mmHg}{ }^{32}$

A neuro exam and MRI may be useful $^{56}$ to differentiate between glaucoma and other types of optic neuropathy. ${ }^{55}$ However, neuroopthalmic evaluation with neuroimaging does not seem to be necessary for all cases of suspected NTG. ${ }^{31}$

NTG is fundamentally the same disease as $\mathrm{POAG}^{31}$, and the treatment is the same, ${ }^{33}$ but some features may be more prevalent in NTG than HTG $^{31,32}$ (splinter haemorrhages for example and CCT typically around 510 to 520 microns). ${ }^{18}$

The collaborative NTG study showed that lowering the IOP by $30 \%$ did change the course of the disease in a majority of patients. ${ }^{10,31,33}$

It is believed that non-IOPrelated factors are more important in NTG, ${ }^{30}$ but recent findings may put that reasoning into question. The SRBD1 gene, which was considered to be a non-IOP-related genetic factor, is implicated in both NTG and HTG. ${ }^{30}$ That HTG and NTG run in the same families suggests they either are the same or are related conditions. ${ }^{31}$ A large proportion of glaucoma patients in Japan suffer from NTG. ${ }^{37}$ (In a Japanese study in the city of Tajimi, $92 \%$ of POAG suffers had NTG. $)^{33}$ There is no global consensus on the question of whether NTG and HTG are simply subtypes of POAG. ${ }^{32,56}$

\section{Diagnostic Techniques}

Stereoscopic ONH photography is a simple low-cost three-dimensional image of the $\mathrm{ONH} .{ }^{10}$ In practice, it is an accepted way of documenting structural damage in glaucoma suspects. ${ }^{15}$

An advantage of subjective assessment over quantitative analysis is the inclusion of some parameters of the $\mathrm{ONH}$ (pallor and haemorrhages) which contribute to a comprehensive evaluation and are not quantifiable. ${ }^{3,38}$ The $\mathrm{ONH}$ has a wide range of normal variations; qualitative variables have been shown to have higher specificity than quantitative parameters in separating glaucomatous eyes from normal. ${ }^{3}$

Assessment of the iridocorneal angle by gonioscopy is key to ruling out a secondary cause. . $15,16,57^{2}$

For decades, Goldmann tonometry has been the most accepted way of measuring IOP. ${ }^{19}$ Goldmann assumed average corneal thickness of $0.52 \mathrm{~mm} .{ }^{19}$ That assumption may lead to either under- or overestimations of IOP with either excessively thin or excessively thick corneas. ${ }^{10,19,31}$

\section{Advances in Diagnostic} Techniques - CCT

OHTS's discovery that lower than average CCT was a risk factor for glaucoma converted pachymetry into a routine procedure in glaucoma assessment. $2,19,35,36,50$

In both OHTS and EGPS, CCT was the most important predictive factor for conversion from $\mathrm{OH}$ to glaucoma. ${ }^{35,36,47,58}$ CCT is no less important in glaucoma suspects with "normal" IOP. In those cases, factors unrelated to pressure are important determiners of progression. ${ }^{19}$

CCT as a risk factor is independent of IOP and possibly based on biomechanical characteristics of the eye. ${ }^{47}$ CCT is not only important because of the artefact of Goldmann's assumption ${ }^{10,31}$ but because patients with thinner corneas also have thinner nerve fibre layers. ${ }^{47}$

Conversion to the "true" pressure based on pachymetry is no longer advocated because there is no consensus on the conversion formula. ${ }^{16,47}$ (Conversion formulae are widely believed to be oversimplifications of a complex nonlinear multifactoral relationship. ${ }^{19,50}$ Instead of converting, we now classify: either thick, average or thin. ${ }^{50}$ Average CCT is approximately $545 \mu \mathrm{m} \cdot{ }^{16,47}$ African Americans generally have thinner corneas, ${ }^{19,50}$ Caucasians' corneas tend to be of average thickness, and Asians' corneas tend to be thicker. ${ }^{36,47}$ The OHTS study highlighted that, after adjustments were made for larger baseline cup-todisk ratios, central corneal thickness and not race was a statistically significant predictor. ${ }^{36}$ 
Recent studies have found that there is considerable variation in the pachymetry readings measured by trained observers. ${ }^{35,47}$ Therefore, pachymetry should be repeated twice, and three times if the first two readings are different. ${ }^{35,47} \mathrm{CCT}$ also changes over the course of the day and over the course of a lifetime, ${ }^{35}$ the cornea is thickest in the morning due to corneal oedema and also thins with age. ${ }^{35}$

\section{Imaging Technology and Retinal} Nerve Fibre Analysis

A new generation of objective (quantitative) imaging technologies have been developed to measure RNFL that go beyond photography, which generally requires subjective interpretation. ${ }^{11,37,39,54}$ To help the clinician in the evaluation of visual function and structure, computerbased imaging devices such as scanning laser ophthalmoscopy (HRT), scanning laser polarimetry (GDx) and optical coherence tomography (OCT) provide quantitative assessment of structural damage to the optic disk and retinal nerve fibre layer. $3,7,9,10,11,15,16,37,41,59,60$

HRT (Heidelberg retina tomograph) is the commercially available form of confocal scanning laser ophthalmoscopy. ${ }^{13,38}$ HRT uses a laser to scan the retina at multiple focal planes and creates a stack of 64 coronal planes. ${ }^{38}$ This provides the examiner with optic disc topography, ${ }^{38}$ a three-dimensional composite image of the $\mathrm{ONH}$ and posterior segment. ${ }^{3,9,38,59}$ HRT can superimpose baseline and follow-up images for automated detection of $\mathrm{ONH}$ changes, and existing machines may easily be upgraded with newer software. ${ }^{3,9}$ The new generation has a higher scan rate and resolution ${ }^{59}$ and eliminates a subjective component by eliminating the need for a contour line drawn by the examiner. ${ }^{3,38,59}$ Scanning laser polarimetry (GDx) uses polarized light to measure the retinal nerve fibre layer birefringence in order to estimate tissue thickness. ${ }^{7,54,39}$ Media opacities decreases its reliability but the newer versions can compensate for this more effectively. ${ }^{3}$ OCT employs the principles of low-coherence interferometry and is analogous to ultrasound B-mode imaging but using light - not sound - to acquire high-resolution images of ocular structures. ${ }^{711,54,59}$ The main limitations of time-domain OCT are low resolution and slow scan rate. ${ }^{40}$ The newest OCT technology - spectral domain ${ }^{40}$ - collects all backscattered light frequencies simultaneously. ${ }^{54}$ That results in a faster scan rate ${ }^{40}$ and better resolution, ${ }^{40}$ with each frequency of light representing a tissue depth. ${ }^{54}$ A new parameter was also introduced for the OCT: signal strength (seven or greater is needed for good quality). ${ }^{59}$ Ocular opacities can decrease the reliability of OCT as well. ${ }^{3}$

Multiple studies have found structural imaging technology to be at least as good as stereo photos in their ability to help clinicians differentiate between glaucomatous and normal eyes. ${ }^{3,37,38,59,60}$ Studies have not demonstrated the clear superiority of one imaging technique over another. ${ }^{3,41}$

It is important to note that the definition of "normal" varies with each technology (HRT, OCT, GDx). ${ }^{59}$ In one study, both GDx VCC and stratus OCT had similar correlations at each clock-hour segment, and both were useful in early detection for patients with preperimetric glaucoma. ${ }^{7}$

In another study, the diagnostic imaging techniques outperformed subjective assessment of the optic nerve by general opthalmolgists (but not by glaucoma specialists, whose expertise outperformed objective techniques)..$^{59}$ All scans had better sensitivity and specificity than general ophthalmologists' subjective photo assessement of ONH. ${ }^{59}$

In several studies, HRT was as effective as stereophotos in estimating risk of developing POAG in ocular hypertensive subjects. ${ }^{3,38,60}$ Therefore, HRT can be used to successfully differentiate ocular hypertensives, normals and eyes with glaucoma. ${ }^{3,9,38,60}$

The use of scanning technology in concert with subjective assessment by either a general ophthalmologist or a glaucoma expert improved identification of glaucoma patients. ${ }^{59}$ This suggests that, particularly for general ophthalmologists, measurement of RNFL provides an important degree of additive information when combined with subjective assessment. ${ }^{41,54,59}$ Scanning technology should not replace observation of the optic nerve (or perimetry) but complement it. ${ }^{11,39,54,59}$

\section{Visual Field Testing}

Despite advances in glaucoma diagnosis, visual field (VF) testing is still important. ${ }^{3,5,34,43,44}$ It is critical to include measurements both of structure and of function in the evaluation of glaucoma although they may not correspond in the early stages of the disease. ${ }^{3,26,34}$ White-on-white static automated perimetry is still the 
most commonly used method to clinically diagnose visual field loss (and monitor progression). 5, 16,37,39,42 It is now suggested that, due to the high variability of results, VF testing be repeated three times to confirm area of loss. ${ }^{3}$ In OHTS, the majority of VF abnormalities initially detected were not repeated on follow-up testing. ${ }^{3}$ SITA-SAP (Swedish interactive threshold algorithm - standard automated perimetry) has become the standard for clinical use with the Humphrey visual field analyzer. ${ }^{3,34}$ SITA is a testing strategy that has decreased testing time to roughly five minutes with no loss of accuracy. ${ }^{3,42}$ GHT (glaucoma hemifield test) analyzes 24-2 and 30-2 visual fields for patterns typically found in glaucoma (such as vertical asymmetry). ${ }^{13}$

Newer technologies like FDT (frequency doubling technology) and SWAP (short wavelength automated perimetry) may be helpful for early detection of disease when SAP is normal but glaucoma is nevertheless suspected. ${ }^{37,42,43}$

FDT perimetry seems to have promise as an effective and efficient method of detecting visual field loss. ${ }^{7,16,43,44}$ It is portable, easier to use and faster than SAP. ${ }^{42}$ FDT loses reliability with cataracts but has lower test-retest variability compared to SAP and SWAP., ${ }^{3,37}$ SWAP is selective and highly sensitive to early damage (up to 5 years earlier than $\mathrm{SAP}^{42}$ ). 3,16 However, like FDT, it is vulnerable to media opacities. ${ }^{3}$

Studies suggested that a combination of field tests may increase sensitivity to early damage without a drop in specificity. ${ }^{42}$ Results of tests can be repeated either within a test or across tests to look for evidence of damage to the same area; $;, 43$ SITA-SAP may be combined with SWAP, for example.

\section{Advances in Therapeutic Options}

Medical treatment with ocular hypotensives is the mainstay of therapy, particularly in the early stages of the disease. ${ }^{1,48}$ Beta-blockers, CAIs and alpha-2 adrenergic agonists decrease formation of aqueous fluid while prostaglandin analogues improve outflow through the uveosceral pathway. ${ }^{2,45,47}$

Prostaglandin analogues (PGAs), the safest and most effective drugs to date, ${ }^{1,47,61}$ have become the preferred first-line agents for glaucoma management. ${ }^{10,46,47}$ Once daily dosing optimizes convenience and increases compliance. ${ }^{6}$ Patients respond differently to each prostaglandin, none of which is universally better than another. ${ }^{47}$

Hyperaemia, which is the most common adverse effect of PGAs (and which has been found to decrease compliance) occurs less frequently in latanoprost. ${ }^{62}$ Timolol (beta-blocker) monotherapy produces far less hyperaemia but is less effective than latanoprost and bimotoprost. ${ }^{1}$ Out of the PGAs, latanoprost (which is associated with less hyperaemia) may achieve the best balance of IOP efficacy and tolerability. ${ }^{1,47}$ There is no clear choice of adjunctive therapy with prostaglandins. Other classes are relatively inefficacious with them. ${ }^{61}$

Contrary to the widely held belief, IOP is actually highest at night..$^{33,47}$ This is likely due to elevated episcleral venous pressure which causes a backup in the drainage system. ${ }^{47}$ Prostaglandins are better at prevent- ing nocturnal spikes (due to mechanism of action) while beta-blockers probably have poor IOP control at night. ${ }^{47}$

Studies have shown Travatan's and Travatan Z's duration of action exceeds their 24-hour dosing schedule (up to 84 hours). ${ }^{46}$ The incidence of IOP varying with missed doses, which has been associated with glaucoma progression, thus decreases. ${ }^{16,46,48}$

Travatan $Z$ is the only PGA that contains SofZia and not BAK as a preservative. ${ }^{46,47} \mathrm{BAK}$ reduces TBUT and decreases epithelial cell integrity, and that causes dry eye and ocular surface inflammation. ${ }^{46,47}$ One study found ocular surface disease is a problem in almost half of all glaucoma patients. The reduction of BAK may be important in glaucoma management. ${ }^{46,47}$ The chronic subclinical inflammation can cause surgical failure in those who have filtration surgery. ${ }^{46,47} \mathrm{BAK}$-free Travatan was found to be at least as effective as original Travatan but better for ocular surface disease. ${ }^{46}$

\section{Management}

The goal of glaucoma management is to protect the patient's vision and quality of life. ${ }^{34}$

IOP reduction should be at least 20 to $30 \%$ lower than where damage occurred. ${ }^{1,16,22}$ The greater the initial IOP, the greater the target reduction. ${ }^{1}$ Achieving these objectives may require several medications, particularly when reduction over $20 \%$ is required. ${ }^{50}$

Determining the effectiveness of IOP-lowering drugs is complicated by a diurnal fluctuation of up to $5 \mathrm{mmHg}$ in normal populations with no evidence of pathology. ${ }^{1}$ Greater 
IOP fluctuations means greater risk of progression. ${ }^{16,34}$ The more the IOP is lowered, the more likely that the glaucoma progression will be stopped. ${ }^{16}$

When pharmacological therapy either fails or is not an option, various surgical procedures are available either to increase outflow or decrease inflow. ${ }^{10,63}$ Laser trabeculoplasty (incision-free) stimulates the trabecular meshwork into functioning more efficiently. ${ }^{2,13,16}$ Laser trabeculoplasty was found to be at least as efficacious as initial treatment with topical medication in the Glaucoma Laser Trial. ${ }^{63}$

Trabeculectomy is used when nonincisional techniques fail. ${ }^{2,16}$ Drainage implants, which are alternatives to incisional techniques, shunt aqueous humor to the subconjunctival space (usually reserved for complicated cases when trabeculectomy fails)., 216 Filtration surgery with cataract removal is not as effective as filtration surgery alone. Patients who need both may want to delay cataract removal. ${ }^{16}$

\section{Adherence}

Adherence to medications is a serious problem, ${ }^{15,16}$ with rates of noncompliance with glaucoma meds sometimes soaring to $80 \%$ of cases in the literature. ${ }^{1,48}$ Lack of consistency in dosing can lead to either sustained increases or fluctuations in levels of IOP. ${ }^{16}$ In either case, the risk of glaucoma progression is increased. ${ }^{16,46}$ Non-compliance may occur for many reasons. The cost of medication may be prohibitive for the patient; the patient may not be able to properly administer dosage. $^{16,48}$ Side effects may dissuade a patient, and a patient may simply fail to understand the importance of treatment and quit. ${ }^{16,48}$ The most common reason cited is forgetfulness. ${ }^{48} \mathrm{Up}$ to $50 \%$ of patients discontinue chronic medications during the first five months of therapy despite its importance in stopping the progression of glaucoma. ${ }^{62}$

The GAPS study showed that $37 \%$ of patients experiencing hyperaemia with PGA will discontinue treatment. ${ }^{1}$ Uninterrupted use of glaucoma medication is relatively rare but is better with latanoprost than with other PGAs. ${ }^{62}$

Electronic monitoring is currently the most objective method to track patient adherence. ${ }^{48}$

\section{The Future of POAG Diagnosis and Management}

A broadly accepted definition of $\mathrm{POAG}^{24,26,27}$ in addition to a universal and cost-effective screening protocol is clearly needed to improve diagnosis and treatment. ${ }^{6}$ Detailed studies on the time-frame from disease onset to significant vision loss and blindness need further investigation. ${ }^{51}$

Most definitions of glaucoma are based on visual field loss but recently a definition incorporating progressive optic disk change has been suggested. ${ }^{3,27}$

Better hardware and software are needed to improve the diagnostic value of imaging technologies ${ }^{11}$ and automated perimetry. ${ }^{43} \mathrm{Fu}-$ ture imaging technology includes polarization-sensitive OCT, which will allow more precise measurement of the RNFL. ${ }^{16,54}$ The next generation of OCT, swept source (SS-OCT), will be even faster than spectral domain and will minimize motion artefacts. ${ }^{54}$ The high cost of imaging technology promotes the economic feasibility of general ophthalmologists receiving ongoing training in optic disc glaucomatous signs, particularly in developing countries. ${ }^{59}$ Current tonometers supply merely a snapshot, but future models may be able to deliver, via implant in the eye, 24 hours of data (as with a Holter moniter). ${ }^{22}$ A new front in the fight against glaucomatous damage may be opened by drugs working through an alternative to IOP-reduction. ${ }^{33,61}$ In particular, drugs which offer neuroprotection (which shield the optic nerve from damage) would be revolutionary. ${ }^{12,14,20,61}$

Communication between optometrists and ophthalmologists needs to be improved; research shows that both the letters of referral to specialists and the responses to ODs leave much to be desired. ${ }^{28}$ A recent study showed that alarms, both audio and visual, boast improved compliance and a decreased proportion of missed doses. ${ }^{48}$ Activities consistent with overall health (such as exercise, a diet high in fruits and vegetables and the avoidance of smoking) may be suggested despite the present lack of conclusive evidence. ${ }^{49}$ Lifestyle and environment are factors requiring further study. They may modify IOP-risk and decrease the economic and visual consequences of POAG. ${ }^{49,51}$

\section{Conclusion}

While much progress has occurred in the diagnosis and management of POAG, the exact pathogenesis and a cure remain elusive. This case highlights the importance of looking beyond IOP and emphasizes the application of modern technology and therapy in the management of this disease. 


\section{References}

1. Orme M, Collins S, Dakin H, Kelly S, Loftus J. Mixed treatment comparison and meta-regression of the efficacy and safety of prostaglandin analogues and comparators for primary open-angle glaucoma and ocular hypertension. Curr Med Res Opin. 2010 Mar;26(3):511-28.

2. Hazin R, Hendrick AM, Kahook MY. Primary open-angle glaucoma: diagnostic approaches and management. J Natl Med Assoc. 2009 Jan;101(1):46-50.

3. Sharma P, Sample PA, Zangwill LM, Schuman JS. Diagnostic tools for glaucoma detection and management. Surv Ophthalmol. 2008 Nov;53 Suppl1:S17-32.

4. Allingham RR, Liu Y, Rhee DJ. The genetics of primary open-angle glaucoma: a review. Exp Eye Res. 2009 Apr;88(4):837-44.

5. Carreras FJ, Rica R, Delgado AV. Modeling the patterns of visual field loss in glaucoma. Optom Vis Sci. 2011 Jan;88(1):63-79.

6. Ghaffariyeh A, Honarpisheh N, Heidari MH, Puyan S, Abasov F. Brain-derived neurotrophic factor as a biomarker in primary open-angle glaucoma. Optom Vis Sci.2011 Jan;88(1):80-5.

7. Kim HG, Heo H, Park SW. Comparison of scanning laser polarimetry and optical coherence tomography in preperimetric glaucoma. Optom Vis Sci. 2011 Jan;88(1):124-9.

8. Lee KJ, Jeong SM, Hoehn BD, Hong YJ, Lee SH. Valosin-containing protein is a novel autoantigen in patients with glaucoma. Optom Vis Sci. 2011 Jan;88(1):164-72.

9. Balasubramanian M, Bowd C, Weinreb RN, Zangwill LM. Agreement between the Heidelberg Retina Tomograph (HRT) stereometric parameters estimated using HRT-I and HRT-II. Optom Vis Sci. 2011 Jan;88(1):140-9.

10. Friedman NJ Kaiser PK, Pineda R. The Massachusetts eye and ear infirmary illustrated manual of ophthalmology. 3rd Ed. Philadelphia: Elsevier, 2009:483-523.

11. Yoo YC, Park KH. Comparison of optical coherence tomography and scanning laser polarimetry for detection of localized retinal nerve fiber layer defects. J Glaucoma. 2010 Apr-

May;19(4):229-36.

12. Cheung W, Guo L, Cordeiro MF. Neuroprotection in glaucoma: drugbased approaches. Optom Vis Sci. 2008 Jun;85(6):406-16.

13. Kanski, JJ. Clinical ophthalmology: a systemic aproach 7th Ed. Oxford: Elsevier, 2011: 311-399, 785-811.

14. Baltmr A, Duggan J, Nizari S, Salt TE, Cordeiro MF. Neuroprotection in glaucoma - Is there a future role? Exp Eye Res. 2010 Nov;91(5):554-66.

15. American Academy of Ophthalmology Glaucoma Panel. Preferred Practice Pattern ${ }^{\circledR}$ Guidelines. Primary OpenAngle Glaucoma Suspect. San Francisco, CA: American Academy of Ophthalmology; 2010. Available at: www.aao.org/ppp.

16. American Academy of Ophthalmology Glaucoma Panel. Preferred Practice Pattern ${ }^{\circledR}$ Guidelines. Primary OpenAngle Glaucoma. San Francisco, CA: American Academy of Ophthalmology; 2010. Available at: www.aao.org/ppp.

17. Kniestedt C, Punjabi O, Lin S, Stamper RL. Tonometry through the ages. Surv Ophthalmol. 2008 NovDec;53(6):568-91.

18. Ehlers JP, Shah CP. The Wills Eye Manual: office and emergency room diagnosis and treatment of eye disease. 5th Ed. Baltimore: Lippincott Williams \& Wilkins, 2008: 191-225.

19. Lester M, Mete M, Figus M, Frezzotti P. Incorporating corneal pachymetry into the management of glaucoma. J Cataract Refract Surg. 2009 Sep;35(9):1623-8.

20. Danesh-Meyer HV. Neuroprotection in glaucoma: recent and future directions. Curr Opin Ophthalmol. 2011 Mar;22(2):78-86.

21. Jia LY, Tam PO, Chiang SW, Ding N, Chen LJ, Yam GH, Pang CP, Wang NL. Multiple gene polymorphisms analysis revealed a different profile of genetic polymorphisms of primary open-angle glaucoma in northern Chinese. Mol Vis. 2009;15:89-98.

22. Stamper RL. A history of intraocular pressure and its measurement. Optom Vis Sci. 2011 Jan;88(1):16-28.
23. Denniss J, Echendu D, Henson DB, Artes PH. Discus: investigating subjective judgment of optic disc damage. Optom Vis Sci. 2011 Jan;88(1):93-101.

24. Miglior S, Guareschi M, Romanazzi F, Albe E, Torri V, Orzalesi N. the impact of definition of primary openangle glaucoma on the cross-sectional assessment of diagnostic validity of Heidelberg retinal tomography. Am J Ophthalmol. 2005 May;139(5):878-87.

25. Swanson MW. The 97.5th and 99.5th percentile of vertical cup disc ratio in the United States. Optom Vis Sci. 2011 Jan;88(1):86-92.

26. Boland MV, Quigley HA. Evaluation of a combined index of optic nerve structureand function for glaucoma diagnosis. BMC Ophthalmol. 2011 Feb 11;11:6.

27. Harwerth RS, Wheat JL, Fredette MJ, Anderson DR. Linking structure and function in glaucoma. Prog Retin Eye Res. 2010 Jul;29(4):249-71.

28. Lockwood AJ, Kirwan JF, Ashleigh Z. Optometrists referrals for glaucoma assessment: a prospective survey of clinical data and outcomes. Eye (Lond). 2010 Sep;24(9):1515-9.

29. Asrani S, Samuels B, Thakur M, Santiago C, Kuchibhatla M. Clinical Profiles of Primary Open Angle Glaucoma versus Normal Tension Glaucoma Patients: A Pilot Study. Curr Eye Res. 2011 May;36(5):429-35.

30. Mabuchi F, Sakurada Y, Kashiwagi K, Yamagata Z, Iijima H, Tsukahara S. Association between SRBD1 and ELOVL5 gene polymorphisms and primary open angle glaucoma. Invest Ophthalmol Vis Sci. 2011 Jun 28;52(7):4626-9.

31. Anderson DR. Normal-tension glaucoma (Low-tension glaucoma). Indian J Ophthalmol. 2011 Jan;59 Suppl:S97-101.

32. Zhang L, Zhang YQ, Xu L, Yang H, Wu XS. [Is normal-tension glaucoma different from primary open-angle glaucoma.]. Zhonghua Yan Ke Za Zhi. 2011 Feb;47(2):105-108.

33. Shields MB. Normal-tension glaucoma: is it different from primary open-angle 
glaucoma? Curr Opin Ophthalmol. 2008 Mar;19(2):85-8.

34. Gardiner SK, Demirel S, Johnson CA. Perimetric indices as predictors of future glaucomatous functional change. Optom Vis Sci. 2011 Jan;88(1):56-62.

35. Brandt JD, Gordon MO, Beiser JA, Lin SC, Alexander MY, Kass MA; Ocular Hypertension Treatment Study Group. Changes in central corneal thickness over time: the ocular hypertension treatment study. Ophthalmology. 2008 Sep;115(9):1550-1556.

36. Gordon MO, Beiser JA, Brandt JD, Heuer DK, Higginbotham EJ, Johnson CA, Keltner JL, Miller JP, Parrish RK 2nd, Wilson MR, Kass MA. The Ocular Hypertension Treatment Study: baseline factors that predict the onset of primary open-angle glaucoma. Arch Ophthalmol. 2002 Jun;120(6):714-20; discussion 82930.

37. Nomoto H, Matsumoto C, Takada S, Hashimoto S, Arimura E, Okuyama S, Shimomura Y. Detectability of glaucomatous changes using SAP, FDT, flicker perimetry, and OCT. J Glaucoma. 2009 Feb;18(2):165-71.

38. Alencar LM, Bowd C, Weinreb RN, Zangwill LM, Sample PA, Medeiros FA. Comparison of HRT-3 glaucoma probability score and subjective stereophotograph assessment for prediction of progression in glaucoma. Invest Ophthalmol Vis Sci. 2008 May;49(5):1898-906.

39. Alencar LM, Zangwill LM, Weinreb RN, Bowd C, Vizzeri G, Sample PA, Susanna R Jr, Medeiros FA. Agreement for detecting glaucoma progression with the GDx guidedprogression analysis, automated perimetry, and optic disc photography.Ophthalmology. 2010 Mar;117(3):462-70.

40. Rao HL, Zangwill LM, Weinreb RN, Sample PA, Alencar LM, Medeiros FA. Comparison of different spectral domain optical coherence tomography scanning areas for glaucoma diagnosis. Ophthalmology. 2010 Sep;117(9):1692-9.

41. Pueyo V, Polo V, Larrosa JM, Ferreras A, Alias E, Honrubia FM. Ability of different optical imaging devices to discriminate between healthy and glaucomatous eyes. Ann Ophthalmol (Skokie). 2009 Summer;41(2):102-8.

42. Alencar LM, Medeiros FA. The role of standard automated perimetry and newer functional methods for glaucoma diagnosis and follow-up. Indian J Ophthalmol.2011 Jan;59 Suppl:S53-8.

43. Fogagnolo P, Rossetti L, Ranno $S$, Ferreras A, Orzalesi N. Shortwavelength automated perimetry and frequency-doubling technology perimetry in glaucoma. Prog Brain Res. 2008;173:101-24.

44. Johnson CA, Wall M, Thompson HS. A history of perimetry and visual field testing. Optom Vis Sci. 2011 Jan;88(1):8-15.

45. Susanna R Jr, Medeiros FA. The pros and cons of different prostanoids in the medical management of glaucoma. Curr Opin Ophthalmol. 2001 Apr;12(2):149-56.

46. Gross RL, Peace JH, Smith SE, Walters TR, Dubiner HB, Weiss MJ, Ochsner KI. Duration of IOP reduction with travoprost BAK-free solution. J Glaucoma. 2008 Apr-May;17(3):217-22.

47. Sowka J. The four Ps of glaucoma. Rev Optom 2010 Nov 15:66-71.

48. Ho LY, Camejo L, Kahook MY, Noecker R. Effect of audible and visual reminders on adherence in glaucoma patients using a commercially available dosing aid. Clin Ophthalmol. 2008 Dec;2(4):769-72.

49. Pasquale LR, Kang JH. Lifestyle, nutrition, and glaucoma. J Glaucoma. 2009 Aug;18(6):423-8.

50. Rhee DJ. Preventing glaucoma in a highrisk population: impact and observations of the Ocular Hypertension Treatment Study. Arch Ophthalmol. 2009 Feb;127(2):216-8.

51. Weinreb RN, Medeiros F. Risk assessment for glaucoma. Open Ophthalmol J. 2009 Sep 17;3:30-1.

52. Shafi A, Swanson WH, Dul MW. Structure and function in patients with glaucomatous defects near fixation. Optom Vis Sci. 2011 Jan;88(1):130-9.

53. Ocular Hypertension Treatment Study Group; European Glaucoma Prevention Study Group, Gordon MO, Torri V, Miglior S, Beiser JA, Floriani I, Miller JP, Gao F, Adamsons I, Poli D, D’Agostino
RB, Kass MA. Validated prediction model for the development of primary open-angle glaucoma in individuals with ocular hypertension. Ophthalmology. 2007 Jan;114(1):10-9.

54. Townsend KA, Wollstein G, Schuman JS. Imaging of the retinal nerve fibre layer for glaucoma. Br J Ophthalmol. 2009 Feb;93(2):139-43

55. Dumitrica DM, Stefan C. [Normotensive glaucoma]. Oftalmologia. 2008;52(1):31-5.

56. Potop V, Dumitrache M, Ciocalteu A. [Normal tension glaucoma]. Oftalmologia. 2010;54(2):11-4.

57. Alward WL. A history of gonioscopy. Optom Vis Sci. 2011 Jan;88(1):29-35.

58. Elsheikh A, Alhasso D, Gunvant P, Garway-Heath D. Multiparameter correction equation for Goldmann applanation tonometry. Optom Vis Sci. 2011 Jan;88(1):102-12.

59. Vessani RM, Moritz R, Batis L, Zagui RB, Bernardoni S, Susanna R. Comparison of quantitative imaging devices and subjective optic nerve head assessment by general ophthalmologists to differentiate normal from glaucomatous eyes. J Glaucoma. 2009 Mar;18(3):253-61.

60. Weinreb RN, Zangwill LM, Jain S, Becerra LM, Dirkes K, Piltz-Seymour JR, Cioffi GA, Trick GL, Coleman AL, Brandt JD, Liebmann JM, Gordon MO, Kass MA; OHTS CSLO Ancillary Study Group. Predicting the onset of glaucoma: the confocal scanning laser ophthalmoscopy ancillary study to the Ocular Hypertension Treatment Study. Ophthalmology. 2010 Sep;117(9):1674-83.

61. Realini T. A history of glaucoma pharmacology. Optom Vis Sci. 2011 Jan;88(1):36-8.

62. Hahn SR, Kotak S, Tan J, Kim E. Physicians' treatment decisions, patient persistence, and interruptions in the continuous use of prostaglandin therapy in glaucoma. Curr Med Res Opin. 2010 Apr;26(4):957-63.

63. Razeghinejad MR, Spaeth GL. A history of the surgical management of glaucoma. Optom Vis Sci. 2011 Jan;88(1):39-47. 